University of Nebraska - Lincoln

DigitalCommons@University of Nebraska - Lincoln

Faculty Publications, College of Dentistry

Dentistry, College of

October 2005

Dental Hygiene Participation in Managing Incipient and Hidden

Caries

Caren M. Barnes

University of Nebraska College of Dentistry, cbarnes@unmc.edu

Follow this and additional works at: https://digitalcommons.unl.edu/dentistryfacpub

Part of the Other Medicine and Health Sciences Commons

Barnes, Caren M., "Dental Hygiene Participation in Managing Incipient and Hidden Caries" (2005). Faculty Publications, College of Dentistry. 1.

https://digitalcommons.unl.edu/dentistryfacpub/1

This Article is brought to you for free and open access by the Dentistry, College of at DigitalCommons@University of Nebraska - Lincoln. It has been accepted for inclusion in Faculty Publications, College of Dentistry by an authorized administrator of DigitalCommons@University of Nebraska - Lincoln. 


\title{
Dental Hygiene Participation in Managing Incipient and Hidden Caries
}

\author{
Caren M. Barnes, RDH, BS, MS \\ Clinical Research, University of Nebraska Medical Center, College of Dentistry, \\ 40th \& Holdrege, Lincoln, NE 68583-0740 USA; email cbarnes@unmc.edu
}

\begin{abstract}
The essence of dental hygiene practice includes the use of preventive and therapeutic methods for the promotion of good health and to prevent and control oral diseases. Dental hygienists actively promote the prevention of oral diseases through patient education that encourages self-care prevention of dental caries, supplemented by needs-related professional preventive services. Equally significant in the scope of dental hygiene practice are thorough oral examinations for disease detection. Although dental hygienists do not legally or definitively diagnose dental caries, they are responsible for detecting caries at all stages of the disease, recognizing caries risk factors, and planning management strategies. Because the focus of this endeavor is the management of incipient and hidden caries, caries prevention will not be the primary focus of this article. It must be underscored that dental caries prevention is one of the primary goals for ethical, sound, and responsible dental hygiene practice.
\end{abstract}

\section{Dental caries detection}

Visual and tactile examination

The traditional methods of caries detection for dental hygienists have included visual inspection, tactile examination with an explorer, and radiographic examination. These traditional practices are still used in contemporary dental hygiene practice; however, some practices have been altered due to paradigm shifts or new diagnostic equipment.

Given that dental caries is now recognized as an infectious, transmittable disease, it is crucial that the dental hygienist identify lesions as early as possible. Carious lesions begin as demineralization of the enamel surface due 
to biofilm and if detected early these lesions can be remineralized via several ways: from saliva, fluoride ingested in foods and water, and with professionally applied fluoride products.

Before a tactile examination is performed, a visual examination of all tooth surfaces is performed on the teeth to identify early lesions. Visual examinations are aided by the use of compressed air. Some early white lesions are undetectable until dried, when they are revealed as a chalky white area. These lesions are also referred to as "hidden carious lesions," a term used to describe carious lesions that are missed on visual, tactile, and radiographic examination $[1,2]$. Hidden lesions that are only visible on a dry tooth surface are probably located only in the outer enamel. Conversely, a lesion visible on a wet tooth has likely penetrated most of the way through the enamel and maybe into dentin [3]. When detected, these chalky white lesions can often be remineralized with fluoride, which is discussed in another section of this article.

The visual examination is accompanied by the tactile examination, most often performed with a dental explorer. The traditional tactile examination is no longer routine and not recommended [4], due to credible scientific evidence that if a demineralizing surface, know as a white-spot lesion [3], is still intact it can have the potential to remineralize. Picking, scratching, or forcing the tip of an explorer into a white-spot demineralizing surface that is still intact can prevent that lesion from remineralizing. This paradigm shift is supported by the National Institutes of Health Consensus Panel on Diagnosis, Treatment, and Management of Dental Caries that concluded that the use of sharp dental explorers to detect caries adds "little diagnostic information and may be detrimental" [5]. Kidd and Fejerskov [3] suggest that to "jab" a sharp explorer into a lesion to determine if it is "sticky" is likely to "cause a cavity and this will encourage biofilm stagnation and lesion progression." They emphasize that the formation of a cavity is a critical moment clinically because the biofilm is protected in a microcavity and the cavity might have first been created by dentists using explorers. In Europe, use of an explorer to probe carious lesions is even considered unethical [6]. Although there are some dental hygiene practitioners who will want continue to use a dental instrument to detect dental caries, if they elect to do so it has been suggested by Wilkins [7] that they use a blunt periodontal probe and gently run the probe over the surface with no pressure, or the dental explorer can gently be used to remove biofilm or debris to assist with visualization [4]. Stookey [4] suggests the very gentle use of a dental explorer for removal of biofilm and debris, using the least amount of pressure possible. Summarily, when examining the patient for demineralizing surfaces or early cavitated lesions (also referred to as "small carious lesions") that have potential for remineralization, the dental hygienist should be looking for:

- Changes in color and translucency of enamel

- White spot lesions that appear chalky white 
- Breaks in the enamel surface

- Grayish-white or gray discolorations adjacent to marginal ridges and margins of restorations

- Shadowing on proximal surfaces

- Black discoloration (arrested caries)

Once the carious lesion has progressed to the point of grayish or brown discoloration it is key to remember what causes these changes in the color of the enamel. Dentin is a vital tissue that responds to bacteria with sclerosis of the tubules. This sclerosis occurs before the advancing cavity in the enamel reaches the dentin. Once the enamel lesion reaches the dentinoenamel junction, a brownish discoloration appears due to the initial demineralization of the dentin [31 and represents a reaction to the biofilm. At this stage there is still a basis for the dental hygienist to intervene with fluoride to attempt the arrest the lesion and for the patient to take an active role in the responsibility for disruption of the biofilm on a regular basis. Once a brownish discoloration is seen in the tooth surface and professional attempts have been made to remineralize the lesion, it is important to track the lesion to determine if attempts to remineralize have been successful. The lesion can be tracked radiographically or with an alternative method that measures changes in mineral content or measures some parameter of lesion infection or infiltration by bacterial products. Included in the visual and tactile examination of the teeth should be the examination for root caries, especially in older patients. Root surface caries are quite similar to enamel caries in that root surface caries begin as a subsurface demineralization. Unlike enamel caries, the root surface lesion appears softer at an earlier stage due to the hardness of cementum being less than that of enamel. Root caries in cementum, like primary enamel caries, can be arrested with biofilm removal and professional intervention with fluoride application.

When using the visual and tactile examination techniques, it is important for the dental hygienist to examine restorations for recurrent or secondary caries adjacent to restorations. Most secondary lesions are the same as primary caries located at the margin of a restoration. Secondary caries are often localized gingivally near restorations. Like primary lesions, every attempt should be made to remineralize those lesions that have not progressed to the point that they require surgical intervention from the dentist. Again, these lesions must be followed radiographically or with an alternative diagnostic method to determine if attempts to remineralize have been successful.

\section{Radiographic examination}

The use of radiographs to examine teeth and other oral structures for the presence of oral disease remains the "gold standard." The use of radiographs allows for the quantification of bone height, examination of bony structures, and apical radiolucencies. As a part of dental hygiene practice, 
dental hygienists expose and interpret radiographs. Critical to the use of radiographs is good technique, so that the resulting radiographs can be interpreted and the retaking of radiographs avoided, a situation that can be disturbing to patients as it requires additional exposure to radiation energy. Characteristics of acceptable radiographs are provided in Table $1[7,9]$. Common mistakes made in the exposure of radiographs are cone cuts, overlapping of interproximal surfaces, and failure to include the desired tooth structure in the film.

\section{Alternative caries detection methods}

In recent years, more than a few caries detection methods and devices have been developed. The advent of these detection methods is welcomed as traditional caries detection procedures do not allow for the detection of caries until they have progressed through at least the thickness of enamel. Some of these new caries detection methods or devices are so recent that they are not yet marketed to the dental profession, and others have been found to be more practical for research settings than for private practice. These include but are not limited to:

- Alternating current impedance spectroscopy technique (ACIST)

- Computer-aided radiography (CAR)

- Dental digital radiography (DDR)

- Digital imaging fiber-optic transillumination (DIFOTI)

- The DIAGNOdent

- The Diagora image plate system (DIPS)

- Diode laser fluorescence (DLF)

- Electrical conductance fixed frequency $(\mathrm{ECFF})$

- Endoscope filtered fluorescence (EFI)

- Qualitative light-induced laser fluorescence (QLF)

- Visualix high-definition imager, intra-oral sensor technology

Table 1. Characteristics of an acceptable radiograph

\begin{tabular}{ll}
\hline Characteristic & Appearance \\
\hline $\begin{array}{l}\text { Image } \\
\text { to natural }\end{array}$ & All parts of teeth of interest must be shown close \\
distortion & size, with minimal overlap and minimal \\
$\begin{array}{l}\text { Area covered } \\
\text { purposes }\end{array}$ & Sufficient tissue surrounding tooth for diagnostic \\
Density & Proper density for diagnosis \\
Contrast & Proper contrast for diagnosis \\
Definition and sharpness & Clear outline of objects; minimal penumbra \\
\hline
\end{tabular}

Data from Wilkins E. Clinical practice of the dental hygienist. 9th edition. Philadelphia: Lippincott, Williams \& Wilkins; 2004; and White SC, Pharoah MJ. Oral radiology principles and interpretation. 5th edition. St. Louis (MO): Mosby; 2004. 
These modalities share the goal of measuring carious lesions, which occur on a continuous scale of tissue damage that varies from subclinical surface changes to macroscopic cavities, rather than simply detecting them. Notably, the ability to identify changes in a lesion on this continuous scale offers the opportunity for the chance at early intervention for remineralization [8]. The opportunity that these new methods and devices provide for early intervention for potential remineralization of carious lesions is a perfect application for the use of dental hygiene services, especially the application of fluoride and developing caries management and prevention plans for patients. This fact brings to mind the question as to whether it is legal in the United States for dental hygienists to use these methods or devices. A query to all 50 states, the District of Columbia, and Puerto Rico was made for the purposes of this article to determine if any of these new detection methods or devices were prohibited for use by dental hygienists. The results of our query were inconclusive, as most state practice acts have not been updated to include these new methods or devices; however, no jurisdiction responded that dental hygienists were precluded from using them.

Because many of the new detection methods are not generally marketed to dental practices, the methods and devices most likely to be encountered in clinical practice by dental hygienists include computerized digital radiography, quantitative light-induced fluorescence (QLF), the DIAGNOdent that uses infrared laser fluorescence, and the DIFOTI, a transillumination method that uses a digital charge couple device to capture the images.

\section{Digital imaging}

Dental image acquisition for dental examinations and diagnosis has been transformed by digital imaging, also known as computerized digital radiography. Although the use of digital radiography has been more slowly accepted by dentistry than by medicine, there is no doubt that this trend of acceptance is growing and it is only a matter of time before it is found in a majority of dental practices. Digital radiography offers a seamless fit into practices that use computer software for tasks such as appointment scheduling, patient charting, and billing.

Digital radiographic images are created by using the spatial distribution of pixels and the different shades of gray of each of the pixels. In other words, the digital radiographic imaging devices interface with a computer to digitize the digital radiographic image into pixels that are then viewed on a computer monitor Figure 1 [9] illustrates how pixels form the image. Rather than using traditional radiographic film, digital radiographs are taken by using a sensor that is placed in the same location that dental film would be placed. The image can be viewed on a computer monitor, stored, transmitted to a remote site, or printed [7,9]. Digital imaging equipment is also available for taking panographic and cephalometric views extraorally. 


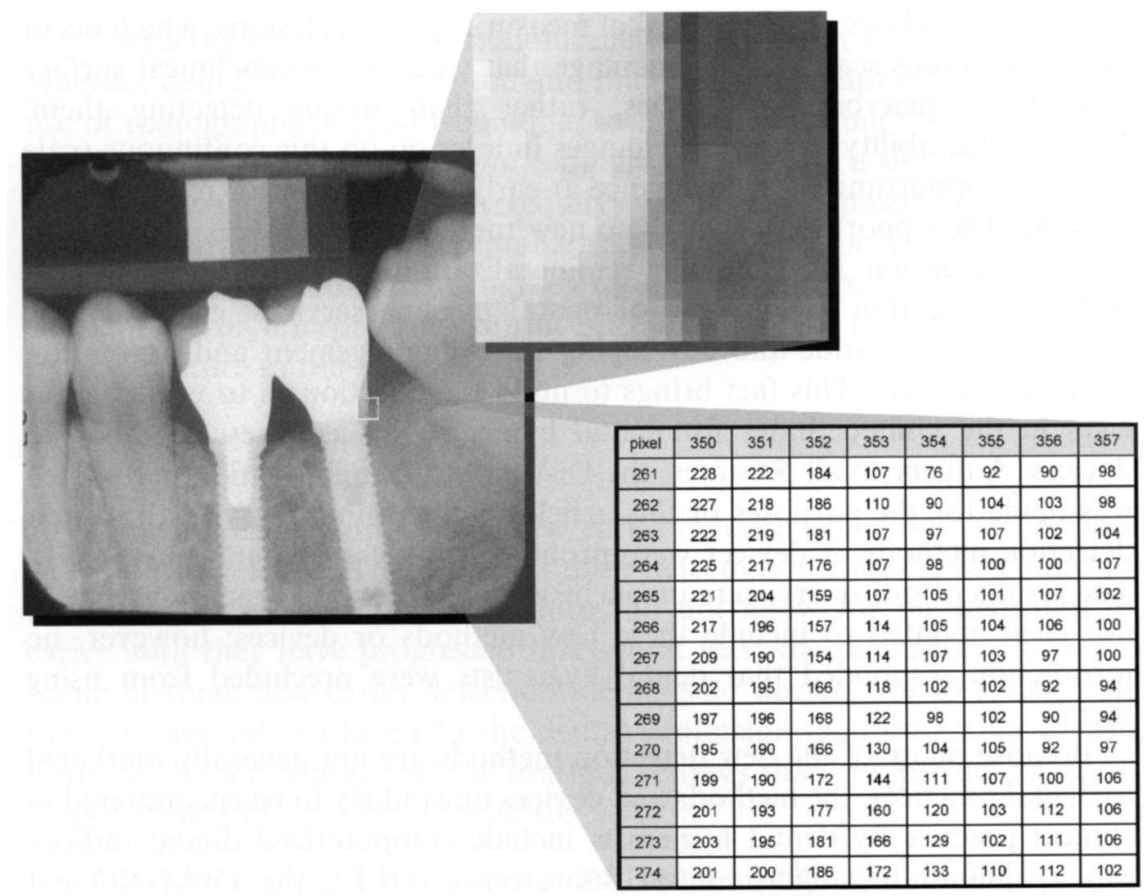

Figure 1. The digital image is made up of a large number of discrete picture elements (pixels). Their size is so small that the image appears smooth at normal magnification. The location of each pixel is uniquely identified by a row and column coordinate within the image matrix. The value assigned to a pixel represents the intensity (gray level) of the image at that location. (From White SC, Pharoah MJ. Oral radiology principles and interpretation. 5th edition. St. Louis (MO): Mosby; 2004. p. 226; with permission.)

The use of dental digital radiographs offers many advantages over traditional dental radiography:

- Radiation exposure to patient is significantly reduced

- Eliminates chemical processing and accompanying errors

- Hazardous waste and lead foil are eliminated

- Same film positioning, no new positioning to learn

- Images can be transferred to other health care professionals without loss of original quality

The most important advantage that digital imaging has for dental hygienists is that is can also be used for subtraction purposes. Images taken over time can be superimposed, which makes an excellent method for tracking bone loss over time in patients with periodontal disease. Likewise, dental hygienists can also use digital subtraction radiography to track hidden and small carious lesions to determine if fluoride applications and patient self-care are being effective in remineralizing these lesions, as more 
than half of shallow dentinal lesions can be arrested and operative therapy avoided [9]. Digital imaging can be used for caries management, not just caries diagnosis.

\section{Quantitative light-induced fluorescence}

Quantitative light-induced fluorescence (QLF) enhances early detection of carious lesions, particularly progression or regression of white spots of smooth surface lesions. QLF uses the intrinsic fluorescence of the teeth within the yellow-green spectrum of visible light [10]. The tooth is illuminated with a blue light that is emitted from a handpiece and causes the tooth structure to fluoresce and the image is captured with a color microvideo charge-coupled device camera. The data are then stored and analyzed by image analysis computer software (Inspektor Dental Care, Amsterdam, The Netherlands). The tooth is seen on the computer monitor as fluorescent green; dark areas indicate mineral loss (Figure 2). With the computer software, the image can be saved and compared over time to track demineralization or remineralization.

Sometimes a red fluorescence appears (Figures 3 and 4), which has an extrinsic source and is emitted by porphyrins metabolized by bacteria in dental biofilm, calculus, or an infected carious lesion and usually indicates a high caries activity. This red fluorescence has a major added benefit for dental hygienists. QLF is a useful method for visualizing dental biofilm that is not visible clinically under ordinary lights. Dental hygienists can use QLF for demonstrating to patients the areas where they have not been successful in self-dental biofilm removal. Thus, QLF serves as an evaluation tool to assess the outcome of preventive self-care plans developed for the patient. Dental hygienists can also use QLF for a very sensitive detection of residual dental biofilm remaining after a professional oral prophylaxis for their own evaluation purposes [10].

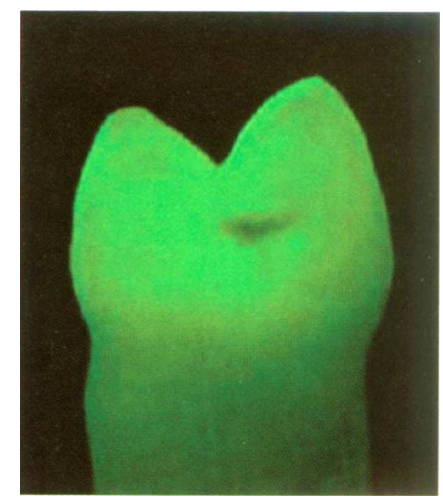

Figure 2. Green fluorescence of the tooth as illuminated with QLF. The dark area indicates mineral loss. (Courtesy of Inspektor Dental Care, Amsterdam, The Netherlands; with permission.) 


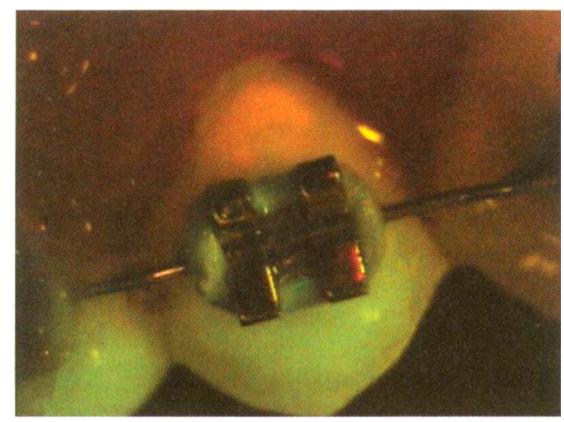

Figure 3. QLF red fluorescence illuminating areas of bacterial activity. (Courtesy of Inspektor Dental Care, Amsterdam, The Netherlands; with permission.)

The red fluorescence is also useful for detecting leaking margins around restorations and leaking margins of sealants. The area of concern can be tracked over time to evaluate the success of remineralization efforts. The dental hygienist should be aware that QLF has a limited ability to detect interproximal lesions [4] and carious lesions that involve two tooth surfaces, as the optical axis of the QLF has to be oriented perpendicular to the tooth surface and the amount of dehydration of a tooth surface can alter the intensity of the fluorescence [10]. At the present time, QLF is being used for research purposes more than for clinical practice. In the very near future, the cost of QLF should be lowered to make it more widely available for private practices [11].

\section{DIAGNOdent}

The DIAGNOdent (KaVo, Lake Zurich, Illinois) also uses fluorescence for caries detection. The DIAGNOdent uses infrared laser fluorescence for the detection of occlusal and smooth surfaces caries. The unit has a fiber-

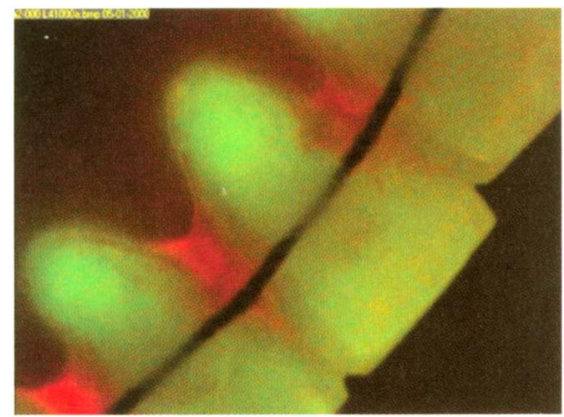

Figure 4. QLF red fluorescence illuminating areas of bacterial activity. (Courtesy of Inspektor Dental Care, Amsterdam, The Netherlands; with permission.) 
optic cable that transmits the light source to a handpiece that contains a fiber-optic eye in the tip. The unit is first calibrated with normal enamel tooth structure in the patient's mouth. After calibration, the handpiece is moved to inspect all surfaces. When the light is absorbed it induces infrared fluorescence of organic and inorganic materials. This fluorescence is collected at the top of the handpiece and transmitted back to the DIAGNOdent unit. The fluorescence is processed, converted into an acoustic signal and displayed as an integer that ranges from 0 to 99 . An increase in fluorescence is indicative of carious tooth substance, particularly when the displayed reading is $\wedge 20$. A reading of 21 to 100 indicates a definite area of decay that may require operative intervention [4]. Most likely, bacterial metabolites, particularly porphyrins, are responsible for production of the fluorescence. According to Stookey [4], the DIAGNOdent is quite useful for the detection of occlusal caries, but he does encourage the use of a dental explorer to remove dental biofilm and debris from occlusal pits and fissures before using the DIAGNOdent. The DIAGNOdent is another alternative caries detection method that can assist the dental hygienist in the early identification of lesions that can be treated by the dental hygienist in a preventive manner rather than by dental surgical intervention.

\section{Digital imaging fiberoptic transillumination}

Transillumination has long been used in dental hygiene for the detection of carious lesions and especially calculus for over 30 years $[4,12]$. The digital imaging fiberoptic transillumination (DIFOTI) system (Figure 5) has elevated traditional transillumination to more sophisticated diagnostic levels. The DIFOTI (Electro-Optical Sciences, Inc., Irvington, New York) allows for images taken from all tooth surfaces to be digitally captured by using

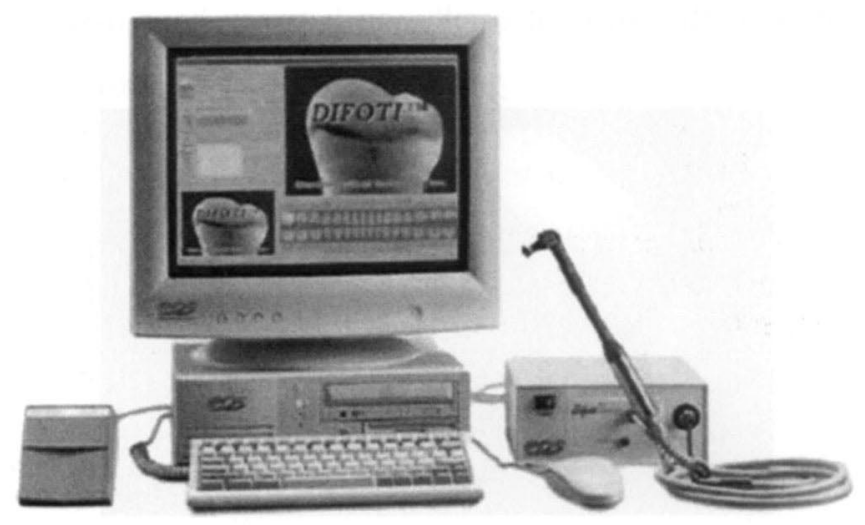

Figure 5. DIFOTI transillumination unit. (Courtesy of Electro-Optical Sciences, Inc., Irvington, New York; with permission.) 
a digital charged-couple device camera and sent to a computer for analysis with dedicated algorithms. The images are stored for longitudinal evaluation of carious lesions [13]. When teeth are transilluminated, the areas of demineralization enamel or dentin scatter light, and incipient caries appear as darker areas in the captured images (Figure 6). The DIFOTI varies from the DIAGNOdent in that it has two handpieces, one for smooth surface caries detection and one for occlusal caries detection. The system uses a disposable mouthpiece and a foot control that allows for selecting the image of concern and a computer for capturing and storing the images. Like the QLF and DIAGNOdent, the DIFOTI can be used for preventive management of early carious lesions, and the information gleaned can be used by the dental hygienist to assist patients in targeting their at-risk-areas. The DIFOTI does not have the capability to determine depths of lesions, but unlike other alternative caries detection devices, it can detect caries on interproximal surfaces as well as radiographs can [4].

\section{Dental hygiene management options for hidden and incipient caries}

Understanding the caries process

Historically, "dental caries" was not treated as an infectious disease and was referred to as a destructive lesion of tooth structure that broke through the tooth structures and created a "cavity" [7]. Recent years have seen a major adjustment in how dental caries is denned. According to the National Institutes of Health, dental caries is now denned as "an infectious, communicable disease resulting in the destruction of tooth structure by acidforming bacteria found in dental biofilm, an intraoral biofilm" [14], and the understanding of the biopathology of dental caries has undergone "major refinement" [15]. A key feature of this understanding that is crucial for the dental hygienist is the constant oscillation between hard-tissue demineral-

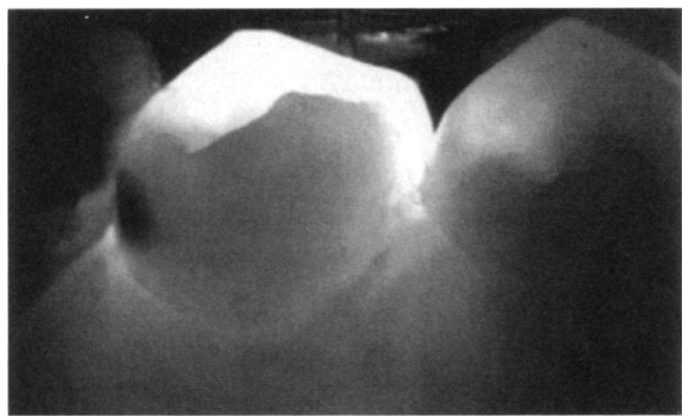

Figure 6. Tooth and carious lesion illuminated with DIFOTI. (Courtesy of Electro-Optical Sciences, Inc., Irvington, New York; with permission.) 
ization and remineralization. Whichever dynamic state the carious lesion is in when detected has a tremendous influence on how the lesion will be managed by the dental hygienist.

\section{Identifying patients at risk}

The initial action taken by the dental hygienist is identifying patients at risk for dental caries. In recent times, many changes in risk identification have occurred. As reported by the National Institutes of Health [14] in 2001, during the past few decades changes have occurred in the incidence, prevalence, distribution, and severity of dental caries. This information can be valuable to the dental hygienist in recognition of patient risk. From an epidemiologic perspective, in many areas of the world the incidence of permanent tooth caries has declined, and this trend is just beginning to emerge in primary tooth caries [15]. A focus on the distribution of dental caries by location in the mouth reveals that occlusal and pit-and-fissure surfaces appear to be increasingly susceptible. With the increasing numbers of older populations retaining their natural dentitions, there is an increasing prominence of root surface caries. As expected within school-aged populations, there is a higher caries incidence in permanent teeth of the lower socioeconomic groups [15].

Awareness of these changes can assist the dental hygienist to accurately predict and identify patients at risk for dental caries and also what types of dental caries patients are at risk for as well as planning management and preventive strategies.

From a patient-focused perspective, the dental hygienist can identify patients at risk for dental caries from comprehensive health, social, and dental histories that assess all three components of caries risk-clinical conditions, environmental characteristics, and general health conditions. Information that is crucial for the dental hygienist to garner from these histories to identify patients at risk for dental caries $[7,16]$ :

- Fluoride history, systemic and topical

- Diet quality, frequency of snacking, types of foods between meals

- Beverage intake, sweetened soda, juices, coffee, tea

- Use of baby bottle for infants

- Gastric reflux or vomiting, eating disorders

- Salivary flow, medications, or conditions that inhibit salivary flow, xerostomia

- Social history, knowledge of dental disease, perceptions of dental needs, value placed on dental health, success in changing habits

- Dental history, sealants, regular dental care, oral hygiene history, caries history

According to the National Institutes of Health Consensus Statement on Dental Caries Management [14], the best indicators for increased risk of den- 
tal caries are: “(I) past experience, especially for assessing children's risk; (2) inadequate exposure to fluoride; (3) any physical or mental illness and any oral application or restoration that compromises the maintenance of optimal oral health; (4) fermentable carbohydrate consumption, (5) lower salivary flow, associated with certain medical conditions and therapies, (6) mutans streptococci; (7) gingival recession, especially in elderly populations; and (8) lower socioeconomic status."

No matter what type of caries risk assessment tool is used, the dental hygienist should gather the information via questionnaire or interview, and the information should be documented in the patient's chart. Two excellent caries-risk assessment tools include the American Academy of Pediatric Dentistry's Caries Risk-Assessment Tool (CAT) [17] and one developed by the American Dental Association Council on Access, Prevention, and International Relations [18]. Caries risk is not stagnant and must be assessed periodically by the dental hygienist to ensure that the patient's caries management plan is needs-based and current. Once caries risk assessment is identified, the dental hygienist can combine this information with information gathered in the comprehensive oral examination to formulate the infrastructure for the patient care plan and education, as well as to initiate the plan for dental caries prevention and management.

\section{Prevention/intervention/therapeutic plan for hidden and incipient caries}

As dental hygienists are primary providers of preventive services, they develop an array of types of preventive plans for patients. The scientific literature is filled with information on these many types of preventive plans and the information required to formulate and implement them. The breadth of this topic is too large to address comprehensively, therefore this section focuses on needs-based patient plans for patients who are susceptible and determined at-risk for incipient and hidden caries.

The disease process of hidden and incipient caries is dynamic: hidden and small carious lesions oscillate between demineralization and remineralization, making it improbable that a preventive plan alone would suffice for the patient if it is a needs-driven plan. In actuality, the dental hygienist is formulating a "prevention/intervention/therapeutic plan" (PITP) as it relates to hidden and incipient caries.

\section{Prevention component}

The prevention portion of the PITP includes the education of the patient, and it is logical to begin the educational portion of the plan with the role of dental biofilm. Patients most likely have heard the term "plaque" and need to know that the term is interchangeable with "dental biofilm." In particular, the patient at-risk for hidden and incipient caries needs to understand two important features of biofilm, one of which is the infectious potential of 
dental biofilm. Parents and caregivers may be surprised to learn that there is evidence of matrilineal transmission of mutans streptococci in infancy and early childhood $[14,19]$. Therefore, the presence of caries in the mother and in siblings can increase the risk for caries in children. The other feature of biofilm that is important for caries at-risk patients to learn is that the ingestion of fermentable carbohydrates increases the destructive nature of biofilm by making it more acidogenic. The consumption of fermentable carbohydrates initiates acid formation and particularly demineralization of tooth structure, particularly in the absence of fluoride. Infants who are on bottles containing fermentable carbohydrates are subject to "baby bottle syndrome," a condition that parents can prevent.

Once the patient has an understanding of dental biofilm and its role in the development of dental caries, the dental hygienist develops the self-care portion of the plan for the patient. The dental hygienist selects brushing and interdental cleaning methods, tongue cleaning, frequency of dental biofilm removal, and selection of oral hygiene aids and dentifrices. Fluoride dentifrices are the most frequently used type of topical fluoride worldwide. Additional decisions include the frequency of recall for oral prophylaxis appointments and professional application of topical fluoride. These decisions are based on the patient's abilities, lifestyle, socioeconomic status, health history, and dental history.

Another principal portion of the self-care prevention plan is patient motivation to change behaviors that place patients at increased risk for caries. In developing the patient's prevention self-care plan, the dental hygienist evaluates the patient's diet to make recommendations for alternatives to fermentable carbohydrates. Other recommendations, such as suggesting the patient use noncariogenic sweeteners like xylitol, can have a large impact on the patient's risk level for hidden and incipient caries. Xylitol is a naturally occurring sugar alcohol, low-calorie sugar substitute that can be found in found in such products as chewing gum, hard candy, and dentifrices. Importantly, xylitol has anticariogenic properties [20], and its use is associated with decreased levels of mutans streptococcus. Many studies of xylitol have been conducted with children in Europe. The findings of these studies reported a consistent decrease in dental caries that ranged from $30 \%$ to $60 \%$ compared with subjects in a control group $[6,21]$. For patients who are at-risk but have no current detectable caries activity, a prudent prevention plan includes the periodic application of topical fluoride or the placement of dental sealants. Topical fluoride is the most important means of dental caries prevention and control. The widespread use of fluoride in dentifrices is no doubt responsible for the worldwide decrease in dental caries [6]. The cariostatic effect of fluoride dentifrices has been recognized for more than 30 years, and currently more than $90 \%$ of dentifrices in industrialized countries contain fluoride. Unfortunately that only accounts for approximately $10 \%$ of the world's population that has access to or uses fluoride-containing 
dentifrices [6]. The results of decades of fluoride research indicate that the caries preventive effect of topical fluorides ranges between $20 \%$ and $40 \%$. Fluoride reduces the demineralizing acids of cariogenic bacteria in dental biofilm and accelerates remineralization. The National Institutes of Health has determined that topical application of acidulated phosphate fluoride once or twice a year and the placement and maintenance of the integrity of pit and fissure sealants are two of the best methods for the primary prevention of dental caries throughout life [17]. Topical fluorides provide an additional benefit when used with fluoridated dentifrices [22].

The type of fluoride selected for use very much depends on patient conditions and should be carefully selected by the dental hygienist. Acidulated phosphate fluoride is contraindicated for patients with hypersensitivity due to the high acid content $(\mathrm{pH}=3.5)$ as is stannous fluoride ( $\mathrm{pH}=2.4$ to 2.8). Both acidulated phosphate fluoride and stannous fluoride are contraindicated for patients who have dental implants as the acid levels are incompatible with implant materials. Acidulated phosphate fluoride is also contraindicated for use on patients who have composites, porcelain, or sealants present as it can etch these materials [23]. Sodium fluoride $(\mathrm{pH}==7)$ is the only type of fluoride that can be used universally and is the most widely used fluoride worldwide. Sodium fluoride and sodium monofluorophosphate are the most commonly used type of fluorides in dentifrices [7]. The application of topical fluorides is not time-consuming, however, it is critical for the dental hygienist to administer the application properly. If tray delivery is used, it is important that the tray fit properly for adequate coverage, and it is imperative that treatments be timed at 4 minutes for maximum uptake.

Patients at-risk for hidden and incipient caries can have their caries risk increased because of the anatomy of pits and fissures, as it is well documented that the configuration of pits and fissures can be a significant risk factor for occlusal caries. The use of the QLF and DIAGNOdent are excellent devices to demonstrate the dental biofilm and debris in these pits and fissures. The biofilm will fluoresce red with the QLF and most likely will produce a reading of $20>$ on the DIAGNOdent display. As pit and fissure caries is biofilm related, age should not be a consideration for dental sealants. Research indicates that occlusal caries susceptibility does not diminish throughout life, therefore, adults can benefit from dental sealants as can children [24]. Some dental professionals are reticent to place dental sealants even though their efficacy is strongly supported in the scientific literature. One of the reasons cited for not placing sealants includes fear of sealing existing decay, despite the fact that numerous studies have demonstrated that bacteria become nonviable and caries does not progress when sealants are applied [25-27]. The sealants eliminate the nutrient sources for mutans streptococcus, which make the lesion inactive [28]. During the annual session of the American Dental Association in 2004, the House of Delegates re- 
ferred three sealant-related resolutions for further study to the Councils on Access, Prevention, and Interprofessional Relations and Scientific Affairs. These resolutions discuss "the effectiveness of dental sealants in preventing pit and fissure dental caries when used as a part of a preventive program and the evidence for using sealants to arrest or manage early carious lesions" [29].

The role of professional oral prophylaxis cannot be overlooked when developing the preventive portion of a PITP. Many dental professionals associate oral prophylaxis on a regular basis with the prevention of periodontal disease. The value of an oral prophylaxis in preventing hidden and incipient caries is just as important. In a study by Axellson and Lindhe [30], a group of school children received professional mechanical tooth cleaning (polishing only) and oral hygiene instruction 16 times per year for 2 years and then four to six times per year for the next 2 years, compared with the control group who received "regular dental care." During the 4-year period, the control group developed 15 times more new carious surfaces than did the test group.

\section{Intervention/therapeutic components}

The intervention/therapeutic components are formulated if the patient has hidden or incipient caries present, thus making the intervention/ therapeutic components of the PITP action-oriented. Once these hidden and small carious lesions are detected, the dental hygienist places great emphasis on the importance of the patient's compliance with self-care measures, especially oral hygiene instruction and dietary modification concomitant with appropriate professional interventions. No amount of preventive intervention or therapeutic services can overcome the neglect of self-care on the part of the patient. The intervention/therapeutic components can include a multitude of treatments that are selected for the patient's specific needs. Because all of these treatments and interventions can not be included in this article, the three treatments focused on in this section are fluoride varnishes, chlorhexidine, and self-applied fluorides.

Fluoride varnishes have been available in Europe for more than 30 years [6], and varnishes have replaced topical gel treatments in many countries [31]. The American Dental Association recently passed a resolution promoting the use of fluoride varnishes at their annual session in October 2004. The American Dental Association "supports the use of fluoride varnishes as safe and efficacious within a caries prevention program that includes caries diagnosis, risk assessment, and regular dental care." The resolution also directs the American Dental Association to "encourage the Food and Drug Administration to consider approving professionally applied fluoride varnish for reducing dental caries, based on the substantial amount of available data supporting the safety and effectiveness of this indication" [29]. 
Research studies have shown fluoride varnishes to be clinically effective, particularly in permanent teeth [32-35]. Research of the effect of fluoride varnishes on caries reduction in primary teeth has not been well-documented to date (however, current research indicates promising results) $[31,36]$. Some of the reasons that fluoride varnishes have been so widely accepted in Europe include ease and safety of the procedure, convenience, and patient acceptance, which are the same reasons that fluoride varnishes appeal to dental hygienists. Additionally, from the dental hygienist's perspective when comparing the venues for administering a tray versus varnish application, the patient is at a reduced risk for fluoride overingestion, and the varnish is easier to apply [37]. The most common types of fluoride varnishes contain sodium fluoride. Duraphat $(5.0 \% \mathrm{~F})$ has been widely studied and a meta-analysis of eight clinical trials investigating the anticaries effect of this fluoride varnish revealed at $35 \%$ reduction in caries [37]. To date, there has only been one direct comparison of the effectiveness of fluoride varnish and acidulated phosphate fluoride gel, and the fluoride varnish was determined to be as effective as gel for caries prevention [34].

Self-applied fluorides for patients are an excellent method of choice for patients to prevent hidden and incipient caries but also to use in conjunction with professionally applied fluorides to arrest hidden and incipient caries that are present. Self-applied fluorides are available in both prescription and over-the counter products and are provided in array of dispensing methods, so it is easy to find a product that patients will accept. Over-the-counter self- applied fluoride products include dentifrices, mouth rinses, and gels and can be delivered traditionally, or a custom tray can be fabricated for delivery. The types of fluoride found in self-applied fluoride products include stannous, neutral sodium, and acidulated phosphate fluorides.

As stated previously, the recommendation for use of a fluoridated dentifrice is the core of a preventive self-care plan for patients. The types of fluoride contained in contemporary brands of dentifrices include sodium fluoride $(0.24 \% ; 1100 \mathrm{ppm})$ and sodium monofluorophosphate $(0.76 \% ; 1000$ ppm or 1500 ppm for an "extra-strength" version). Dentifrices are a continuing source of fluoride and are essential for the control of demineralization and promotion of remineralization. Numerous research studies have demonstrated that the incidence of dental caries can be reduced $20 \%$ to $30 \%$ when a fluoridated dentifrice is used.

Fluoride mouth rinses contain differing types of fluoride than dentifrices. Mouth rinses contain one of the three types of fluoride with the following concentrations:

- Acidulated phosphate fluoride $0.044 \%$

- Neutral sodium fluoride $0.05 \%$

- Stannous fluoride $0.63 \%$ 
Generally, in the concentrations listed previously, patients are advised to use the mouth rinses for 1 minute and then expectorate.

Self-applied fluoride gels are available over-the-counter or by prescription. Generally, over-the-counter gels contain $0.5 \%$ acidulated phosphate fluoride or $1.1 \%$ sodium fluoride and can be delivered with a toothbrush or a tray. They are important not only for patients with hidden and incipient caries but also for patients with rampant caries, xerostomia, radiation therapy, and hypersensitivity. If acidulate phosphate fluoride is contained in this gel, patients with porcelain, composites, titanium, or sealants should not use them. Some gels that are indicated for brushing on also have a dentifrice added. These gels can include $1.1 \%$ neutral sodium fluoride gels (prescription only) or $0.4 \%$ stannous fluoride that is available over-the-counter.

No matter what type of fluoride product is recommended or whether is it used in a combination of patient self-applied fluorides and professionally applied topical fluorides, this is the most important product a dental hygienist can recommend for patients regarding caries arrest and prevention. Much consideration should be given toward the patient's condition in selecting the appropriate product(s), which includes the types of materials that are present in the patient's mouth. Safety cannot be overlooked. Fluoride has a margin of safety, but patients have to be given explicit instructions regarding dosage and frequency of use.

\section{Summary}

Dental hygiene services are best used when they are applied to prevention and control of hidden and incipient caries. Dental hygiene is the perfect preventive complement to dentistry and for patients can make all the difference in disease outcomes. With the advent of new alternative caries detection methods and myriad choices of fluoride products and sealants, patients can see first-hand the successes that dental hygienists have in reversing hidden and incipient caries and thus can appreciate the essence of preventive and therapeutic services dental hygienists are equipped to deliver.

\section{Acknowledgments}

The author expresses her gratitude to the following people for their research and administrative support during the development of this article: Danielle Ryan, RDH, MS, Rita Charles, Dr. David A. Covey, Dr. David Brown, Julie Layton, and Diane Thompsen.

\section{References}

[1] Ricketts D, Kidd E, Weerheijm K, de Soet H. Hidden caries: what is it? Does it exist? Does it matter? Int Dent J 1996;47(5):259-65. 
[2] Guignon AN. Integration of a laser fluorescence caries detection device in dental hygiene practice. Compend Contin Educ Dent 2004;24(5 Suppl):13-7.

[3] Kidd EA, Fejerskov 0. What constitutes dental caries? Histopathology of carious enamel and dentin related to the action of cariogenic biofilms [special issue]. J Dent Res 2004;83(C): C35-8.

[4] Stookey GK. The evolution of caries detection. Dimensions of Dental Hygiene 2003; October: $12-5$.

[5] Horowitz AM. A report on the NIH consensus development conference on diagnosis and management of dental caries throughout life [special issue]. J Dent Res 2004;83(C): C15-7.

[6] Axelsson P. Diagnosis and risk prediction of dental caries, volume 2. Chicago: Quintessence International; 2000.

[7] Wilkins E. Clinical practice of the dental hygienist. 9th edition. Philadelphia: Lippincott, Williams \& Wilkins; 2004.

[8] Huysmans MC, Longbottom C. The challenges of validating diagnostic methods and selecting appropriate gold standards [special issue]. J Dent Res 2004;83(C):C48-52.

[9] White SC, Pharoah MJ. Oral radiology principles and interpretation. 5th edition. St. Louis (MO): Mosby; 2004.

[10] Weltizen R, Kuhnisch J, van der Veen M, et al. Quantitative light-induced fluorescence (QLF): a potential for the dental practitioner. Quintessence Int 2003;34:181-8.

[11] Pretty IA, Edgar WM, Highham SM. The effect of dehydration on quantitative light induced fluorescence analysis of early enamel demineralization. J Oral Rehab 2004;31(2):179-84.

[12] Eakle WA, Gansky SA, Zhan L, et al. Clinical evaluation of the DIAGNOdent device. In: Stookey GK, editor. Early detection of dental caries. 3rd edition. Indianapolis (IN): Indiana University School of Dentistry; 2004.

[13] Perry IA, Maupome G. A closer look at diagnosis in clinical dental practice: part 5. Emerging technologies for caries detection and diagnosis. J Can Dent Assoc 2004;70(8):540.

[14] Glock M, Horowitz AM, Canto MT, compilers. Diagnosis and management of dental caries. Current bibliographies in medicine 2001-1. Available at: http://www.nlm.nih. gov/pubs/cbm/dental_caries.html. Accessed February 1, 2001.

[15] Pitts NB, Stamm JW. Preface. Proceedings from the international consensus workshop on caries clinical trials [special issue]. J Dent Res 2004;83(C):C4.

[16] Kandray DP. Caries risk and nutrition in adolescents. Journal of Practical Hygiene 2004; 13(5): 19-22.

[17] American Academy of Pediatric Dentistry Council on Clinical Affairs. Policy statement on the use of a caries-risk assessment tool, adopted 2002. Available at: http://www. aapd.org/pdf/policycariesriskassessmenttool.pdf

[18] Reithman J. Trends in preventive care: caries risk assessment and indications for sealants. J Am Dent Assoc 2000;131(6):8S-12S.

[19] Caufield PW, Cutter GR, Dasanayake AP. Initial acquisition of mutans streptococci by infants: evidence for a discrete window of infectivity. J Dent Res 1993;72(1):37-45.

[20] Lynch H, Milgrom P. Xylitol and dental caries: an overview for clinicians. J Calif Dent Assoc 2003;31(3):205-9.

[21] Hayes $C$. The effect of non-cariogenic sweeteners on the prevention of dental caries: a review of the evidence. J Dent Educ 2001;65(10):1106-9. 
[22] Monopoli M. Topical fluorides provide additional benefit when used with fluoride toothpaste. Evid Based Dent 2004;5(2):38.

[23] Soeno K, Matsumura H, Atsuta M, Kawasaki K. Influence of acidulated phosphate fluoride agents and effectiveness of subsequent polishing on composite material surfaces. Oper Dent 2002;27(3):305-10.

[24] Ripa L, Leske G, Varma A. Longitudinal studies of the caries susceptibility of occlusal and proximal surfaces of first permanent molars. J Public Health Dent 1988;48:8-13.

[25] Handelman S. Microbiologic aspects of sealing carious lesions. J Prev Dent 1976;3(2):2932. [26] Going R, Loesche W, Grainger D, et al. The viability of microorganisms in carious lesions five years after covering with a fissure sealant. JADA 1978;97:455-62.

[27] Kramer P, Zeiante F, Simionato M. The immediate and long-term effects of invasive and non-invasive pit and fissure sealing techniques on the microflora in occlusal fissures of human teeth. Pediatr Dent 1993;15:108-12.

[28] Mertz-Fairhurst E, Schuster G, Fairhurst C. Arresting caries by sealants: results of a clinical study. JADA 1986; 112:194-7.

[29] Crozier S. ADA House OKs fluoride varnishes. ADA News. November 16, 2004. Available at: http://www.ada.org/prof/resources/pubs/adanews/adanewsarticle. asp?articleid $=1157$

[30] Axelsson P. An introduction to risk prediction and preventive dentistry. Chicago; Quintessence International; 1999.

[31] Autio-Gold JT, Courts F. Assessing the effect of fluoride varnish on early carious lesions in the primary dentition. J Am Dent Assoc 2001;132(9):1247-53.

[32] Ogaard B, Seppa L, Rolla G. Professional topical fluoride applications: clinical efficacy and mechanism of action. Adv Dent Res 1994; 8(2): 190-201.

[33] Skold L, Sundaquist B, Eriksson B, et al. Four-year study of caries inhibition of intensive Duraphat application in 11-to 15-year-old children. Community Dent Oral Epidemiol 1994; 22:8-12.

[34] Seppa L, Leppanen T, Hausen H. Fluoride varnish versus acidulated phosphate fluoride gel: a 3-year clinical trial. Caries Res 1995;29:327-30.

[35] Zimmer S, Robke FJ, Routlet JF. Caries prevention with fluoride varnish in a socially deprived community. Community Dent Oral Epidemiol 1999;27:103-8.

[36] Grodzka K, Augustyniak L, Budny J, et al. Caries increment in primary teeth after application of Duraphat fluoride varnish. Community Dent Oral Epidemiol 1982;10:55-9.

[37] Hawkins R, Locker D, Noble J, Kay EJ. Prevention Part 7: Professionally applied topical fluorides for caries prevention. Br Dent J 2003; 195(6):313-7. 